\title{
A Presença de Patógenos Respiratórios no Biofilme Bucal de Pacientes com Pneumonia Nosocomial ${ }^{*}$
}

\author{
Presence of Respiratory Pathogens in the Oral \\ Biofilm of Patients with Nosocomial Pneumonia
}

\author{
Luiz Cláudio Borges Silva de Oliveira', Pedro Paulo Martins Carneiro², \\ Ricardo Guimarães Fischer ${ }^{3}$, Eduardo Muniz Barreto Tinoco ${ }^{4}$
}

\section{RESUMO}

JUSTIFICATIVA E OBJETIVOS: O paciente internado em unidade de terapia intensiva (UTI), geralmente apresenta higiene bucal insatisfatória, podendo a região orofaríngea ser colonizada por patógenos envolvidos em pneumonia nosocomial. O objetivo deste estudo foi investigar a presença de patógenos respiratórios na cavidade bucal em pacientes em UTI.

MÉTODO: Foram incluídos neste estudo transversal, 30 pacientes residentes no município de Nova Friburgo no estado do Rio de Janeiro, com idade entre 18 e 82 anos e média ponderada de 53,53 anos, sendo 17 homens e 13 mulheres, internados na UTI geral, excetuando a unidade coronariana, do Hospital Municipal Raul Sertã, Nova Friburgo, com diagnóstico de pneumonia nosocomial (PN). Foi realizada cultura das amostras do aspirado traqueal para identificar os micro-organismos

1. Cirurgião-Dentista Especialista e Mestre em Periodontia pelo Centro de Pesquisas Odontológicas São Leopoldo Mandic, Campinas, SP. Doutorando em Periodontia pela UERJ.

2. Médico Especialista em Medicina Intensiva pela AMIB. Presidente da Comissão de Controle de Infecção Hospitalar do Hospital Municipal Raul Sertã em Nova Friburgo, RJ.

3. Médico e Cirurgião-Dentista, Professor Titular de Periodontia da UERJ; Diretor do Instituto de Odontologia da PUC - RJ; Doutor em Periodontia pela Universidade de Lund - Suécia.

4. Cirurgião-Dentista. Doutor pela Universidade de Oslo, Noruega. Professor Adjunto de Periodontia da UERJ/UNIGRANRIO.

*Recebido da Unidade de Terapia Intensiva do Hospital Municipal Raul Sertã, Nova Friburgo, RJ

Apresentado em 30 de outubro de 2007

Aceito para publicação em 11 de dezembro de 2007

Endereço para correspondência:

Dr. Luiz Cláudio Borges Silva de Oliveira

Av. Sernambetiba, 3300, B8/902 Barra da Tijuca

22630-010 Rio de Janeiro, RJ

E-mail: dr.luizclaudioborges@yahoo.com.br

(C)Associação de Medicina Intensiva Brasileira, 2007 responsáveis pela PN. Em contrapartida amostras microbiológicas da placa dental supragengival, da língua e do tubo do umidificador, foram analisadas para avaliação da presença do agente etiológico da PN.

RESULTADOS: As bactérias mais freqüentemente encontradas no aspirado traqueal dos pacientes foram $S$. pneumoniae $23,3 \%$ (7), P. aeruginosa $20 \%$ (6), S. aureus 13,3\% (4), Kleibsella pneumoniae 13,3\% (4), Candida albicans $6,6 \%$ (2), Streptococcus $\alpha$-hemolítico 6,6\% (2), Staphylococcus sp. 6,6\% (2), Acinetobacter calcoaceticus - baumanii complex (A. calcoaceticus) em 1 paciente (3,3\% do total de pacientes), Eschericia coli (E.coli) 3,3\% (1), Enterobacter cloacae (E. cloacae) 3,3\% (1). Nesses pacientes, $70 \%$ destas bactérias foram encontradas no biofilme dental, $63,33 \% \mathrm{em}$ amostras da língua, 73,33\% nas amostras do tubo do respirador artificial e em $43,33 \%$ em todos as áreas simultaneamente. Não foram observadas diferenças significativas nas proporções das amostras dos locais de coleta $(p>0,05)$.

CONCLUSÕES: Os resultados deste estudo sugeriram que a presença de patógenos respiratórios no biofilme bucal de pacientes internados em UTI pode servir de reservatório para micro-organismos associados com pneumonia nosocomial.

Unitermos: Biofilme Oral, Medicina Periodontal, Pneumonia Nosocomial.

\section{SUMMARY}

BACKGROUND AND OBJECTIVES: Hospitalized patients receiving treatment at intensive care units (ICU) usually show poor oral hygiene, and may have the mouth and oropharingeal region colonized by pathogens involved in nosocomial pneumonia. The presence of these pathogens may increase the risk for respiratory diseases. The aim of this study was to investigate the presence of respiratory pathogens in the oral cavity 
of hospitalized patients at ICU.

METHODS: Were included in the study 30 patients from Hospital Raul Sertã, Nova Friburgo, with the diagnostic of nosocomial pneumonia, and tracheal aspirate samples were cultured to identify the causing microorganisms. In addition, microbiological samples from supragingival dental plaque, tongue and respiratory tube were cultured for the presence of a panel of respiratory pathogens.

RESULTS: The most frequently found bacteria in the tracheal aspirate were S. Pneumoniae $23.3 \%$ (7), P. aeruginosa $20 \%$ (6), S. aureus $13.3 \%$ (4), K. pneumoniae $13.3 \%$ (4), C. albicans $6.6 \%$ (2), $\alpha$-hemolytic streptococcus $6.6 \%$ (2), Staphylococcus sp. $6.6 \%$ (2), A. calcoaceticus $3.3 \%$ (1), E. coli $3.3 \%$ (1) and E. cloacae $3.3 \%$ (1). $70 \%$ (21) of these microorganisms were found in the dental biofilm, $63.33 \%$ (19) in tongue samples; $73.33 \%$ (22) in the respiratory tube; and $43.33 \%$ (13) in all sampling sites simultaneously. No differences in proportions could be observed between the sampling sites $(p>0.05)$

CONCLUSIONS: The results of this study show that respiratory pathogens associated with nosocomial pneumonia are present in the oral biofilm of hospitalized patients in ICU, which may serve as a reservoir for these microorganisms.

Key Words: Oral Biofilm, Periodontal Medicine, Nosocomial Pneumonia.

\section{INTRODUÇÃO}

A pneumonia é um acometimento no parênquima pulmonar causada por grande variedade de agentes, incluindo bactérias, micoplasma, fungos, parasitas e vírus ${ }^{1}$, sendo a pneumonia bacteriana a causa mais comum da doença².

A pneumonia é usualmente classificada em pneumonia adquirida na comunidade ou pneumonias nosocomiais, que são aquelas desenvolvidas após $48 \mathrm{~h}$ de internação hospitalar e que não estavam presentes ou incubadas no paciente no momento da admissão no hospital ${ }^{3}$.

Dentre todas as infecções adquiridas em hospital, a pneumonia nosocomial é responsável por 10\% a 15\% deste total; e $20 \%$ a $50 \%$ de todos os pacientes afetados por infecções falecem. O risco de desenvolvimento de pneumonia nosocomial é de 10 a 20 vezes maior na unidade de terapia intensiva (UTI), sendo que o seu desenvolvimento em pacientes com ventilação mecânica e/ou umidificador varia de $7 \%$ a $40 \%$. Estimativas indicam que nos EUA mais de 300.000 infecções respiratórias nosocomiais ocorram a cada ano, resultando em 20.000 mortes/ano e gastos aproximados de US\$ 30 bilhões em cuidados hospitalares e antibioticoterapia com estes pacientes ${ }^{4,5}$.

Existem duas formas para os micro-organismos bucais alcançarem o trato respiratório inferior: difusão hematogênica e aspiração ${ }^{6-8}$. A difusão hematogênica das bactérias é rara e só há dois casos documentados na literatura ${ }^{9,10}$. Em contrapartida, a aspiração de microorganismos originários das vias aéreas superiores durante o sono ocorre em $45 \%$ dos pacientes saudáveis e em $70 \%$ dos pacientes com a percepção prejudicada, tais como alcoólatras, usuários de drogas, epilépticos ${ }^{11}$. Os micro-organismos podem contaminar o trato respiratório inferior através de quatro possíveis vias: 1) aspiração do conteúdo da orofaringe ${ }^{7}$; 2 ) inalação de aerossóis infectados ${ }^{1}$; 3) disseminação da infecção através de áreas contíguas ${ }^{12}$; 4) disseminação hematogênica através de áreas infecciosas extrapulmonares (p. ex.: infecção do trato gastrintestinal) ${ }^{13,14}$. Assim, pode ser sugerido que há três mecanismos possíveis para se associar o biofilme bucal com infecções respiratórias. Primeiro, o biofilme bucal com higiene deficiente ${ }^{15-17}$, resultaria em alta concentração de patógenos na saliva, que poderiam ser aspirados para o pulmão em grandes quantidades, deteriorando as defesas imunes ${ }^{18-21}$. Segundo, através de condições específicas, o biofilme bucal poderia abrigar colônias de patógenos pulmonares e promover seu crescimento ${ }^{22-24}$. Por fim, as bactérias presentes no biofilme bucal poderiam facilitar a colonização das vias aéreas superiores por patógenos pulmonares ${ }^{12,14}$.

O objetivo deste estudo foi investigar a possível presença de patógenos respiratórios na cavidade bucal em pacientes internados em UTI.

\section{MÉTODO}

Após aprovação do Comitê de Ética em Pesquisa da Universidade do Estado do Rio de Janeiro (UERJ), foi realizado o estudo em conjunto com a equipe médica da UTI do Hospital Municipal Raul Sertã (HMRS), seguindo o protocolo clínico do hospital.

Durante os meses de janeiro a novembro de 2007 deram entrada na UTI do HMRS 128 pacientes, e neste universo, foram incluídos neste estudo transversal, 30 pacientes residentes no município de Nova Friburgo no estado do Rio de Janeiro, com idade entre 18 e 82 anos e média ponderada de 53,53 anos, sendo 
17 homens e 13 mulheres, internados na UTI geral, excetuando a unidade coronariana do hospital, com diagnóstico de pneumonia nosocomial (PN). Todos os pacientes estavam com umidificadores, não utilizando no momento da coleta, respiradores artificiais. Foram colhidas amostras para cultura com swab estéril do aspirado traqueal, baseado na rotina laboratorial do $\mathrm{HMRS}^{25}$ para identificar as bactérias responsáveis pela $\mathrm{PN}$ após $48 \mathrm{~h}$ de internação e diagnóstico clínico da $\mathrm{PN}^{26}$. Todos os pacientes internados na UTI foram examinados pela equipe médica intensivista e a coleta de dados dos prontuários realizada pelo mesmo pesquisador calibrado. Os critérios de exclusão foram pacientes edentado total; episódio prévio de aspiração do conteúdo gástrico; presença de monitor de pressão intracraniana, cirurgia torácica e doença pulmonar obstrutiva crônica; portadores do vírus da síndrome da imunodeficiência adquirida (SIDA).

Foram considerados pacientes com pneumonia nosocomial todos aqueles que apresentaram todos os sinais clínicos após 48 horas de internação na UTI. O diagnóstico realizado pela equipe médica intensivista, para pneumonia nosocomial, constituiu de temperatura corporal maior do que $38^{\circ} \mathrm{C}$ ou menor que $36^{\circ} \mathrm{C}$; presença de infiltrado evidenciado na radiografia de pulmão; leucocitose (> $\left.10 \times 10^{3} / \mathrm{mm}^{3}\right)$; cultura positiva na aspiração traqueal $\left(>10^{6} \mathrm{ufc} / \mathrm{mL}\right)^{27}$. A coleta nas áreas foi realizada pelo mesmo pesquisador, imediatamente após a confirmação do diagnóstico de PN, antes da administração de antibioticoterapia.

Nestes pacientes selecionados para o estudo, foram coletadas amostras com swab estéril nos seguintes locais: o biofilme no dorso de língua, a superfície dos primeiros molares superiores, na ausência desses foi analisado $\circ 1^{\circ}$ dente posterior ou anterior, no tubo do umidificador e a cultura do aspirado traqueal. As seguintes bactérias foram encontradas nos três locais examinados: Pseudomonas aeruginosa, Staphylococcus aureus, Kleibsella pneumoniae, Stenotrophomas maltophilia, Candida albicans, Acinetobacter calcoaceticus - baumanii complex, Streptococcus ( $\alpha$-hemolítico grupo viridans), Candida tropicalis, Staphylococcus sp., Serratia marcescens, Actinobacter calcoaceticus bio anitratus, Corynebacterium sp., Eschericia coli, Streptococcus pneumoniae, Enterobacter cloacae. A coleta foi realizada pelo mesmo pesquisador calibrado nos pacientes, e as análises microbiológicas realizadas pelo mesmo laboratório.

Foi realizada anamnese pelo pesquisador juntamente com a equipe dos médicos intensivistas para verifica- ção dos fatores de risco para a ocorrência da pneumonia nosocomial.

Os pacientes e/ou responsáveis foram orientados a respeito da pesquisa e, de maneira voluntária, deram seu consentimento, sendo necessário o preenchimento de um formulário TCLE de acordo com a resolução 196/96 - Conselho Nacional de Saúde do Ministério da Saúde.

\section{RESULTADOS}

Dos 128 pacientes que deram entrada na UTI do HMRS, 98 foram excluídos da pesquisa por critérios metodológicos. Nos 30 pacientes selecionados os resultados da análise do aspirado traqueal demonstraram que as bactérias mais freqüentemente encontradas foram: $S$. pneumoniae em 7 pacientes $(23,3 \%)$, sendo no total destes pacientes, em cinco a bactéria estava presente em apenas uma área, sendo em quatro no tubo do umidificador e em um paciente no biofilme dental, e nos outros dois pacientes a bactéria estava presente nos três locais - biofilme dental, biofilme da língua e no tubo do umidificador - ao mesmo tempo da coleta; $P$. aeruginosa em seis pacientes $(20 \%)$ sendo que a bactéria estava presente no tubo do umidificador, em cinco ela estava presente além do tubo do umidificador também no biofilme dental e da língua e em um paciente apenas no tubo do umidificador; $S$. aureus em quatro pacientes $(13,3 \%)$ sendo que em um paciente a bactéria estava presente somente no biofilme da língua, em dois pacientes além do biofilme da língua estavam presentes também nos outros dois locais - tubo do umidificador e biofilme dental - e em um paciente estava presente no tubo do umidificador e no biofilme dental; K. pneumoniae em quatro pacientes $(13,3 \%)$ sendo que em três pacientes a bactéria foi encontrada nos três locais - biofilme dental, biofilme da língua e tubo do umidificador simultaneamente e em um paciente foi encontrada no biofilme dental e da língua; C. albicans em dois pacientes $(6,6 \%)$ sendo em um paciente a bactéria identificada apenas no biofilme da língua e no outro, foi encontrada no biofilme dental e no tubo do umidificador; Streptococcus $\alpha$-hemolítico em dois pacientes $(6,6 \%)$, em um paciente a bactéria foi encontrada no biofilme dental e no biofilme da língua e no outro paciente a bactéria foi identificada apenas no biofilme dental; Staphylococcus sp. em dois pacientes (6,6\%). Em ambos as bactérias foram encontradas no biofilme dental e no biofilme da língua, $A$. calcoaceticus em um paciente $(3,3 \%)$ sendo a bactéria encontrada 


\section{A PRESENÇA DE PATÓGENOS RESPIRATÓRIOS NO BIOFILME BUCAL DE PACIENTES COM PNEUMONIA NOSOCOMIAL}

nos três locais observados - biofilme dental, biofilme da língua tubo do umidificador; $E$. coli em um paciente $(3,3 \%)$ que foi identificada no tubo do umidificador e $E$. cloacae em um paciente $(3,3 \%)$ identificada no tudo do umidificador (Tabela 1).

Dentre as 15 bactérias pesquisadas, Pseudomonas aeruginosa, Staphylococcus aureus, Kleibsella pneumoniae, Stenotrophomas maltophilia, Candida albicans, Acinetobacter calcoaceticus - baumanii complex, Streptococcus $\alpha$-hemolítico (grupo viridans),
Candida tropicalis, Staphylococcus sp., Serratia marcescens, Actinobacter calcoaceticus bio anitratus, Corynebacterium sp., Eschericia coli, Streptococcus pneumoniae, Enterobacter cloacae, duas delas, Acinetobacter calcoaceticus - baumanii complex e Candida tropicalis não foram detectadas nos exames microbiológicos em nenhum dos três locais pesquisados (Tabela 2). Das 12 bactérias identificadas, 10 foram responsáveis pelo desenvolvimento da PN nos pacientes (Tabela 1). Dos 30 pacientes com pneumo-

Tabela 1 - Freqüência e Distribuição das Bactérias Etiológicas da Pneumonia Nosocomial por Local

\begin{tabular}{|c|c|c|c|c|c|c|c|c|}
\hline Bactérias $^{1}$ & $\begin{array}{l}\text { Número de } \\
\text { Pacientes }^{2}\end{array}$ & $\begin{array}{c}\text { Tubo do } \\
\text { Umidificador } \\
\text { (TU) }\end{array}$ & $\begin{array}{c}\text { Biofilme } \\
\text { Dental }^{4} \\
\text { (BD) } \\
\end{array}$ & $\begin{array}{l}\text { Biofilme } \\
\text { da Língua }{ }^{5} \\
\text { (BL) }\end{array}$ & $\begin{array}{c}\mathrm{TU} \\
+ \\
\mathrm{BD}^{6}\end{array}$ & $\begin{array}{c}\mathrm{TU} \\
+ \\
\mathrm{BL}^{7} \\
\end{array}$ & $\begin{array}{c}\mathrm{BD} \\
+ \\
\mathrm{BL}^{8} \\
\end{array}$ & $\begin{array}{c}\mathrm{TU} \\
+ \\
\mathrm{BD}+\mathrm{BL}^{9} \\
\end{array}$ \\
\hline S.pneumoniae & $7(23,3 \%)$ & 4 & 1 & & & & & 2 \\
\hline$P$. aeruginosa & $6(20,0 \%)$ & 1 & & & & & & 5 \\
\hline S. aureus & $4(13,3 \%)$ & & & 1 & 1 & & & 2 \\
\hline K.pneumoniae & $4(13,3 \%)$ & & & & & & 1 & 3 \\
\hline C. albicans & $2(6,6 \%)$ & & & 1 & 1 & & & \\
\hline $\begin{array}{l}\text { Streptococcus } \\
\alpha \text {-hemolítico }\end{array}$ & $2(6,6 \%)$ & & 1 & & & & 1 & \\
\hline Staphylococcus sp. & $2(6,6 \%)$ & & & & & & 2 & \\
\hline A. calcoaceticus & $1(3,3 \%)$ & & & & & & & 1 \\
\hline E. coli & $1(3,3 \%)$ & 1 & & & & & & \\
\hline E. cloacae & $1(3,3 \%)$ & 1 & & & & & & \\
\hline Total & $30(100 \%)$ & 7 & 2 & 2 & 2 & 0 & 4 & 13 \\
\hline
\end{tabular}

1. Bactéria detectada através do aspirado traqueal como agente etiológico da PN.

2. $\quad N^{\circ}$ de pacientes com PN com etiologia bacteriana identificada através do aspirado traqueal.

3. $\quad \mathrm{N}^{\circ}$ de pacientes com bactéria somente no tubo do umidificador (TU).

4. $\quad \mathrm{N}^{\circ}$ de pacientes com bactéria somente no biofilme dental (BD).

5. $\quad \mathrm{N}^{\circ}$ de pacientes com bactéria somente no biofilme da língua (BL).

6. $\quad N^{\circ}$ de pacientes somente com bactéria no TU concomitante ao BD.

7. $\quad N^{\circ}$ de pacientes somente com bactéria no TU concomitante ao BL.

8. $\mathrm{N}^{\circ}$ de pacientes somente com bactéria no BD concomitante ao $\mathrm{BL}$.

9. $\quad N^{\circ}$ de pacientes com bactéria no TU, BD e BL

Tabela 2 - Freqüência da Identificação das Bactérias nos Pacientes da Unidade de Terapia Intensiva

\begin{tabular}{|c|c|c|c|c|c|c|c|c|}
\hline Bactérias $^{1}$ & $\begin{array}{l}\text { Número de } \\
\text { Pacientes }^{2}\end{array}$ & $\begin{array}{c}\text { Tubo do } \\
\text { Umidificador } \\
(\mathrm{TU})^{3}\end{array}$ & $\begin{array}{l}\text { Biofilme } \\
\text { Dental } \\
(B D)^{4} \\
\end{array}$ & $\begin{array}{c}\text { Biofilme } \\
\text { da Língua } \\
(\mathrm{BL})^{5}\end{array}$ & $\begin{array}{c}\text { TU } \\
+ \\
\mathrm{BD}^{6}\end{array}$ & $\begin{array}{c}\mathrm{TU} \\
+ \\
\mathrm{BL}^{7} \\
\end{array}$ & $\begin{array}{c}\mathrm{BD} \\
+ \\
\mathrm{BL}^{8} \\
\end{array}$ & $\begin{array}{c}\mathrm{TU}+ \\
\mathrm{BD}+ \\
\mathrm{BL}^{9} \\
\end{array}$ \\
\hline S.pneumoniae & 10 & 5 & 1 & 1 & - & - & 1 & 2 \\
\hline$P$. aeruginosa & 11 & 1 & 2 & - & - & 1 & 2 & 5 \\
\hline S. aureus & 15 & 2 & 1 & 1 & 3 & 1 & 2 & 5 \\
\hline K.pneumoniae & 11 & - & 1 & 1 & - & - & 5 & 4 \\
\hline C. albicans & 11 & 1 & 2 & 3 & 1 & 1 & 3 & - \\
\hline Streptococcus & 6 & - & 2 & - & 1 & - & 2 & 1 \\
\hline$\alpha$-hemolítico & & & & & & & & \\
\hline Staphylococcus sp. & 10 & - & 1 & 3 & - & 1 & 4 & 1 \\
\hline A. calcoaceticus baumanii complex & - & - & - & - & - & - & - & - \\
\hline E. coli & 8 & 2 & 1 & 1 & - & 1 & - & 3 \\
\hline E. cloacae & 3 & 1 & - & 2 & - & - & - & - \\
\hline S. maltophilia & 1 & 1 & - & - & - & - & - & - \\
\hline C. tropicalis & - & - & - & - & - & - & - & - \\
\hline S. marcescens & 3 & 1 & 1 & 1 & - & - & - & - \\
\hline Corynebacterium sp & 3 & - & - & 3 & - & - & - & - \\
\hline A. calcoaceticus bio anitratus & 2 & - & - & 1 & - & - & - & 1 \\
\hline
\end{tabular}

1. Bactérias identificadas na análise laboratorial.

2. $\quad \mathrm{N}^{\circ}$ de pacientes contaminados com a bactéria.

3. $\quad N^{\circ}$ de pacientes com bactéria somente no tubo do umidificador (TU).

4. $\quad N^{\circ}$ de pacientes com bactéria somente no biofilme dental (BD).

5. $\quad N^{\circ}$ de pacientes com bactéria somente no biofilme da língua (BL).

6. $\quad N^{\circ}$ de pacientes somente com bactéria no TU concomitante ao BD.

7. $\quad N^{\circ}$ de pacientes somente com bactéria no TU concomitante ao BL.

8. $\mathrm{N}^{\circ}$ de pacientes somente com bactéria no BD concomitante ao BL.

9. $\quad \mathrm{N}^{\circ}$ de pacientes com a bactéria no TU, BD e BL. 
nia nosocomial, em dois pacientes $(6,6 \%)$ a etiologia bacteriana da pneumonia nosocomial confirmada no aspirado traqueal mostrou que a mesma bactéria estava presente apenas no biofilme dental, em dois pacientes $(6,6 \%)$ elas estavam presentes apenas na língua, em sete pacientes apenas no tubo do umidificador $(23,33 \%)$; em dois pacientes $(6,6 \%)$ a bactéria estava presente ao mesmo tempo no tubo do umidificador e biofilme dental; em 4 pacientes $(13,2 \%)$ a bactéria estava presente concomitantemente no biofilme dental e língua; e em 13 pacientes $(43,33 \%)$ em todos os locais examinados (Tabela 1). Foram utilizados os testes de Qui-quadrado e Exato de Fisher para diferenças entre proporções para avaliação estatística e nenhuma diferença nas proporções pôde ser observada entre os locais examinados $(p>0,05)$.

\section{DISCUSSÃO}

O objetivo deste estudo foi avaliar a possibilidade de micro-organismos responsáveis pela etiologia da pneumonia nosocomial estarem presentes no biofilme da cavidade bucal e orofaríngea do paciente internado na UTI.

Desde as décadas de 1970 e 1980, alguns autores identificaram bactérias presentes no biofilme dental que poderiam colonizar a cavidade orofaríngea ${ }^{24,28,29}$. Os resultados deste estudo sugerem a possibilidade de que as bactérias responsáveis pela pneumonia nosocomial nos pacientes internados na UTI do HMRS podem ser provenientes do seu biofilme bucal. Estes resultados corroboram com os dados da pesquisa realizada por Scannapieco e col. ${ }^{21}$ sobre a colonização bacteriana do biofilme dental em pacientes internados em UTI. Em outro estudo Fourrier e col. ${ }^{27}$ observaram que, após cinco dias de internação em UTI, os pacientes que desenvolveram pneumonia nosocomial tinham sua etiologia bacteriana associada com a composição bacteriana da placa dental.

Patógenos respiratórios não são usualmente encontrados na flora indígena bucal de pacientes saudáveis ${ }^{21}$. Nesta linha, os resultados bacteriológicos deste estudo são similares com achados de outros estudos ${ }^{21,31}$.

Em contrapartida, patógenos respiratórios originários do meio ambiente hospitalar, podem colonizar as superfícies dos dentes, próteses, mucosa bucal e respectivo biofilme $e^{1,21,27}$. O estabelecimento de patógenos respiratórios exógenos nestas superfícies pode ser facilitado pela diminuição parcial, ou total, da salivação e do $\mathrm{pH}$ da saliva, fatos estes encontrados em pacientes de UTI devido à medicação administrada ${ }^{32}$. As fontes possíveis destes micro-organismos abrangem os procedimentos que envolvem fluídos como aspiração nasogástrica ${ }^{33}$, uso de nebulizadores e umidificadores ${ }^{34}$, alimentação ${ }^{35}$ e manipulação do paciente pela equipe intensivista envolvida em seu tratamento ${ }^{6}$.

Um fator determinante para ocorrer a colonização bacteriana é sua adesão às superfícies. Estudos in vitro já demonstraram desde os anos 1970 e 1980 que bactérias como Pseudomonas aeruginosa e Klebsiella pneumoniae se aderem com mais facilidade as células epiteliais de pacientes hospitalizados do que aqueles não hospitalizados ${ }^{35,36}$. Algumas pesquisas demonstraram uma relação inversa entre a quantidade de fibronectina proveniente das células epiteliais e aderência de bactérias Gram-negativas ${ }^{37,38}$. A sua diminuição pode aumentar a quantidade de receptores para adesinas bacterianas ${ }^{1}$ e a saliva contém enzimas hidrolíticas que diminuem o poder de adesão dos receptores às adesinas bacterianas ${ }^{39,40}$. Entretanto, uma higienização bucal inadequada concomitante ao uso de fármacos, irá alterar a capacidade de salivação, assim como seu pH. Desta forma, promoverão um aumento da quantidade de bactérias no biofilme bucal, que degradarão a fibronectina presente na mucosa, através da produção de enzimas $^{24,27}$, imunoglobulina $A_{1}{ }^{41}$ e glicoproteínas ${ }^{42}$ durante seu metabolismo.

Estes dados sugerem que enzimas bacterianas provenientes do biofilme bucal promovam uma alteração bioquímica da superfície oral do paciente, aumentando desta forma a adesão e conseqüentemente sua colonização por patógenos respiratórios ${ }^{43}$. A ampla variedade destes patógenos respiratórios encontrado no resultado da pesquisa realizada no biofilme bucal dos pacientes internados na UTI do HMRS sugerem, em consenso com dados de outras pesquisas ${ }^{21,27}$, que provavelmente houve alteração na distribuição de receptores localizados no epitélio da superfície da mucosa oral.

O resultado deste estudo suscita a alta probabilidade de colonização de patógenos respiratórios no biofilme bucal de pacientes internados em UTI, onde $70 \%$ das bactérias pesquisadas foram encontradas somente no biofilme dental dos pacientes, sendo $63,33 \%$ destas mesmas bactérias encontradas na língua.

De acordo com os resultados deste estudo, a colonização do biofilme bucal de pacientes em UTI por patógenos respiratórios sugere ser mais um fator de risco relacionado com o desenvolvimento de pneumonia nosocomial nos pacientes estudados. 


\section{CONCLUSÃO}

Os resultados desta pesquisa sugerem que a cavidade bucal de pacientes internados em UTI pode servir como importante reservatório para patógenos respiratórios associados à pneumonia nosocomial. Estes dados propõem uma nova visão em que procedimentos específicos para o controle destes patógenos na cavidade oral devem ser considerados na prevenção de pneumonia nosocomial, especialmente em pacientes de UTI.

\section{AGRADECIMENTOS}

Agradecemos ao corpo clínico da UTI, a comissão de controle de infecção hospitalar $(\mathrm{CCIH})$ e ao laboratório do HMRS, pela assistência técnica laboratorial fornecida durante este estudo.

\section{REFERÊNCIAS}

1. Toews GB - Nosocomial pneumonia. Am J Med Sci, 1986;291:355-367.

2. The committee for the Japanese Respiratory Society guidelines in management of respiratory infections. Respirology, 2004;9:S3-S5

3. Lode H, Raffenberg M, Erbes R et al - Nosocomial pneumonia: epidemiology, pathogenesis, diagnosis, treatment, and prevention. Curr Opin Infect Dis, 2000;13:377-384.

4. Craven DE, Steger KA, Barber TW - Preventing nosocomial pneumonia: state of the art and perspectives for the 1990s. Am J Med, 1991;91:44S53S.

5. Kollef MH, Fraser VJ. Antibiotic Resistance in the intensive care unit. Ann Intern Med 2001;134:298-314

6. Finegold SM - Aspiration pneumonia. Rev Infect Dis, 1991;13:(Suppl9):S37-S742.

7. Sinclair DG, Evans TW - Nosocomial pneumonia in the intensive care unit. Br J Hosp Med, 1994;51:177-180.

8. The committee for The Japanese Respiratory Society guidelines in management of respiratory infections. Respirology 2004;9:S30-S34.

9. Morris JF, Sewell DL - Necrotizing pneumonia caused by mixed infection with Actinobacillus actinomycetemcomitans and Actinomyces israelii: case report and review. Clin Infect Dis, 1994;18:450-452.

10. Christensen PJ, Kutty K, Adlam RT et al - Septic pulmonary embolism due to periodontal disease. Chest, 1993;104:1927-1929.

11. Huxley EJ, Viroslav J, Gray WR et al - Pharyngeal aspiration in normal adults and patients with depressed consciousness. Am J Med, 1978;64:564-568

12. Mojon P - Oral health and respiratory infection. J Can Dent Assoc, 2002;68:340-345

13. Bueno-Cavanillas A, Delgado-Rodriguez M, Lopez-Luque A et al - Influence of nosocomial infection on mortality rate in an intensive care unit. Crit Care Med, 1994;22:55-60.

14. Scannapieco FA, Bush RB, Paju S - Associations between periodontal disease and risk for nosocomial bacterial pneumonia and chronic obstructive pulmonary disease. A systematic review. Ann Periodontol, 2003;8:54-69.

15. Socransky SS, Gibbons RJ, Dale AC et al - The microbiota of the gingival crevice area of man. Total microscopic and viable counts and counts of specific organisms. Arch Oral Biol, 1963;8:275-280.

16. Moore WE, Moore LV - The bacteria of periodontal disease. Periodontol 2000, 1994;5:66-77.

17. Contreras $\mathrm{A}$, Slots $\mathrm{J}$ - Herpesviruses in human periodontal disease. $\mathrm{J}$ Periodontal Res, 2000;35:3-16.
18. Manganiello AD, Socransky SS, Smith C et al - Attempts to increase viable count recovery of human supragingival dental plaque. J Periodontal Res, 1977;12:107-109.

19. Socransky SS, Manganiello AD, Propas D et al - Bacteriological studies of developing supragingival dental plaque. J Periodontal Res, 1977; $12: 90-106$

20. Limeback $\mathrm{H}$ - The relationship between oral health and systemic infections among elderly residents of chronic care facilities: a review. Gerodontology, 1988;7:131-137.

21. Scannapieco FA, Stewart EM, Mylotte JM - Colonization of dental plaque by respiratory pathogens in medical intensive care patients. Crit Care Med, 1992;20:740-745.

22. Fuxench-Lopez Z, Ramirez-Ronda CH - Pharyngeal flora in ambulatory alcoholic patients: prevalence of gram-negative bacilli. Arch Intern Med, 1978;138:1815-1816.

23. Scannapieco FA, Mylotte JM - Relationships between periodontal disease and bacterial pneumonia. J Periodontol, 1996;67:(Suppl10):1114-1122.

24. Wikstrom M, Linde A - Ability of oral bacteria to degrade fibronectin. Infect Immun, 1986;51:707-711.

25. Chastre J, Fagon JY, Lamer C - Procedures for the diagnosis of pneumonia in ICU patients. Intensive Care Med, 1992;18:(Supll1)S10-S17.

26. Agvald-Ohman C, Wernerman J, Nord CE et al - Anaerobic bacteria commonly colonize the lower airways of intubated ICU patients. Clin Microbiol Infect, 2003;9:397-405.

27. Fourrier F, Duvivier B, Boutigny $\mathrm{H}$ et al - Colonization of dental plaque: a source of nosocomial infections in intensive care units patients. Crit Care Med, 1998;26:301-308.

28. Gibbons RJ - Bacterial adhesion to oral tissues: a model for infectious diseases. J Dent Res, 1989;68:750-760.

29. Johanson WG, Pierce AK, Sanford JP - Changing pharyngeal bacterial flora of hospitalized patients. Emergence of gram-negative bacilli. N Engl J Med, 1969;281:1137-1140.

30. Johanson WG Jr, Pierce AK, Sanford JP et al - Nosocomial respiratory infections with gram- negative bacilli. The significance of colonization of the respiratory tract. Ann Intern Med, 1972;77:701-706.

31. Komiyama K, Tynan JJ, Habbick BF et al - Pseudomonas aeruginosa in the oral cavity and sputum of patients with cystic fibrosis. Oral Surg Oral Med Oral Pathol, 1985;59:590-594.

32. Ayars GH, Altman LC, Fretwell MD - Effect of decreased salivation and $\mathrm{pH}$ on the adherence of Klebsiella species to human buccal epithelial cells. Infect Immun, 1982;38:179-182.

33. Millership SE, Patel N, Chattopadyay B - The colonization of patients in an intensive treatment unit with Gram negative flora: The significance of the oral route. J Hosp Infect, 1986;7:226-235.

34. Botman MJ, de Krieger RA - Contamination of small-volume medication nebulizers and its association with oropharyngeal colonization. J Hosp Infect, 1987;10:204-208

35. Johanson WG Jr, Woods DE, Chaudhuri T - Association of respiratory tract colonization with adherence of gram-negative bacilli to epithelial cells. J Infect Dis, 1979;139:667-673.

36. Johanson WG Jr, Higuchi JH, Chaudhuri TR et al - Bacterial adherence to epithelial cells in bacillary colonization of the respiratory tract. Am Rev Respir Dis, 1980;121:55-63.

37. Abraham SN, Beachey EH, Simpson WA - Adherence of streptococcus pyogenes, Escherichia coli, and Pseudomonas aeruginosa to a fibronectincoated and uncoated epithelial cells. Infect Immun, 1983;41:1261-1268.

38. Hasty DL, Simpson WA - Effects of fibronectin and other salivary macromolecules on the adherence of Escherichia coli to buccal epithelial cells. Infect Immun, 1987;55:2103-2109.

39. Nakamura M, Slots $\mathrm{J}$ - Salivary enzymes. Origin and relationship to periodontal disease. J Periodont Res, 1983;18:559-569.

40. Zambon JJ, Nakamura M, Slots J - Effect of periodontal therapy on salivary enzyme activity. J Periodont Res, 1985;20:652-659.

41. Frandsen EV, Reinholdt J, Kilian M - Enzymatic and antigenic characterization of Immunoglobulin $\mathrm{A}_{1}$ proteases from Bacteroides and Capnocytophaga spp. Infect Immun, 1987;55:631-638.

42. Marik PE, Careau P - The role of anaerobes in patients with ventilatorassociated pneumonia and aspiration pneumonia: a prospective study. Chest, 1999;115:178-183.

43. Teng YT, Taylor GW, Scannapieco F et al - Periodontal health and systemic disorders. J Can Dent Assoc, 2002;68:188-192. 